\title{
IL-1 mediates TNF-induced osteoclastogenesis
}

\author{
Shi Wei, Hideki Kitaura, Ping Zhou, F. Patrick Ross, and Steven L. Teitelbaum \\ Department of Pathology, Washington University School of Medicine, St. Louis, Missouri, USA.
}

\begin{abstract}
TNF-induced receptor activator NF- $\mathrm{B}$ ligand (RANKL) synthesis by bone marrow stromal cells is a fundamental component of inflammatory osteolysis. We found that this process was abolished by IL-1 receptor antagonist (IL-1Ra) or in stromal cells derived from type I IL-1 receptor-deficient (IL-1RI-deficient) mice. Reflecting sequential signaling of the cytokines TNF and IL-1, TNF induces stromal cell expression of IL-1 and IL-1RI. These data suggest that TNF regulates RANKL expression via IL-1, and, therefore, IL-1 plays a role in TNF-induced periarticular osteolysis. Consistent with this posture, TNF-stimulated osteoclastogenesis in cultures consisting of WT marrow macrophages and stromal cells exposed to IL-1Ra or in cocultures established with IL-1RI-deficient stromal cells was reduced approximately $50 \%$. The same magnitude of osteoclast inhibition occurred in IL-1RI-deficient mice following TNF administration in vivo. Like TNF, IL-1 directly targeted osteoclast precursors and promoted the osteoclast phenotype in a TNF-independent manner in the presence of permissive levels of RANKL. IL-1 is able to induce RANKL expression by stromal cells and directly stimulate osteoclast precursor differentiation under the aegis of p38 MAPK. Thus, IL-1 mediates the osteoclastogenic effect of TNF by enhancing stromal cell expression of RANKL and directly stimulating differentiation of osteoclast precursors.
\end{abstract}

\section{Introduction}

Focal osteolysis, a major complication of conditions such as rheumatoid arthritis, periodontal disease, and orthopedic implant loosening, reflects accelerated bone resorption prompted by proinflammatory cytokines. The osteoclast, which is the cell ultimately responsible for bone destruction, is a polykaryon formed by fusion of mononuclear precursors of the monocyte/macrophage family under the influence of the specific osteoclastogenic cytokine, receptor activator NF-кB ligand (RANKL) (1, 2). Thus, delineating the mechanism of RANKL expression, in the context of inflammation, is central to identifying novel therapeutic targets for prevention of periarticular osteolysis.

Regulation of RANKL expression is key to the pathogenesis of many osteopenic disorders. In fact, the ratio of RANKL to its soluble, antiosteoclastogenic decoy receptor, osteoprotegerin (OPG), is a reasonable indicator of the magnitude of systemic bone loss in these pathological conditions. Thus, molecules capable of accelerating bone resorption, such as parathyroid hormone, exert their osteoclastogenic effects by directly promoting RANKL expression and inhibiting synthesis of OPG (3).

In the context of bone, RANKL is produced by marrow stromal cells and their derivative osteoblasts. Activated T lymphocytes and synovial cells, in states of skeletal inflammation, also express the osteoclastogenic molecule. On the other hand, TNF is probably the dominant cytokine extant in inflammatory osteolysis (4-8). While TNF-induced osteoclastogenesis requires at least constitutive levels of RANKL, a synergistic relationship exists between the 2 cytokines in directly inducing marrow macrophages to commit

Nonstandard abbreviations used: hMSC, human mesenchymal stem cell; IL-1Ra, IL-1 receptor antagonist; IL-1RI, type I IL-1 receptor; OPG, osteoprotegerin; RANKL, receptor activator NF-кB ligand; TRAP, tartrate-resistant acidic phosphatase. Conflict of interest: The authors have declared that no conflict of interest exists. to the osteoclast phenotype (9). This dependency of TNF-mediated osteoclastogenesis on attendant RANKL is underscored by the absence of meaningful osteoclast recruitment or bone destruction in the face of experimental inflammatory arthritis in mice in which the RANKL receptor has been deleted (10).

Like TNF, IL-1, another osteoclastogenic cytokine, promotes RANKL expression by marrow stromal cells and osteoblasts (11). The means by which these cytokines induce RANKL in stromal/ osteoblastic cells are, however, poorly understood. IL-1 and TNF are expressed in abundance in rheumatoid arthritis, and drugs that inhibit one or the other cytokine are presently used in its treatment. For unknown reasons, blockade of either IL-1 or TNF does not completely arrest the periarticular damage of inflammatory arthritis, whereas inhibition of the 2 cytokines in combination is substantially more effective (12-14).

Systemic anti-TNF therapy is attended by potentially fatal complications such as fungal infection and tuberculosis. Hence, much is to be gained by identifying the TNF-responsive cells that mediate the cytokine's pathological properties with the aim of specific therapeutic targeting. Because marrow stromal cells are a prime source of RANKL and thus contribute to the genesis of inflammatory osteolysis, we explored the means by which TNF prompts their production of RANKL and ultimately osteoclastogenesis in vitro and in vivo. We found that TNF induction of RANKL expression by marrow stromal cells is substantially mediated by IL- 1 via enhanced expression of IL-1RI. Like TNF, IL-1 has the capacity to directly target mononuclear osteoclast precursors and promote their differentiation but requires permissive levels of RANKL to do so. Thus, IL-1 is a key downstream effector molecule in optimal TNF-induced osteoclastogenesis, participating in both stimulated RANKL expression by stromal cells and direct targeting of osteoclast precursors.

\section{Results}

TNF induces RANKL gene expression by marrow stromal cells. To establish that TNF specifically induces RANKL expression in our 
A

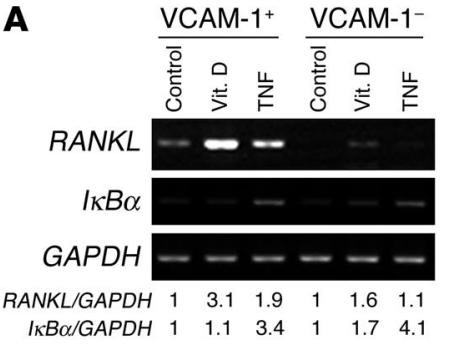

\section{B}

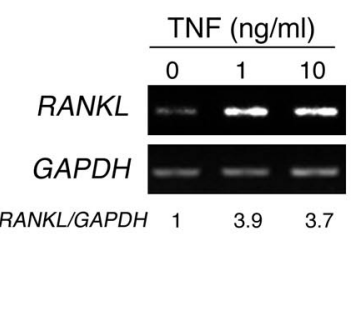

\section{Figure 1}

TNF induces RANKL gene expression by murine and human marrow stromal cells. (A) VCAM-1-positive and -negative murine marrow cells were exposed to PBS (Control), 1,25-dihydroxyvitamin D (Vit. D; $\left.10^{-8} \mathrm{M}\right)$, or TNF (10 $\mathrm{ng} / \mathrm{ml}$ ) for 24 hours. (B) hMSCs were cultured for 1 week and then exposed to 1 or $10 \mathrm{ng} / \mathrm{ml} \mathrm{TNF}$ for 24 hours. In both circumstances, total RNA was extracted and analyzed by RT-PCR. GAPDH mRNA served as loading control. Results are expressed as $A U$ relative to control (arbitrarily set as 1 ). experimental system, we isolated purified populations of primary murine marrow stromal cells on a VCAM-1 affinity column ( 9 , 15). VCAM-1-positive and-negative cells were then exposed to vehicle (control) or TNF for 24 hours. 1,25(OH $)_{2} \mathrm{D}_{3}$, a known transactivator of the RANKL gene, served as positive control. The quantity of RANKL mRNA in equal amounts of total RNA was determined by RT-PCR. As shown in Figure 1A, TNF augments RANKL mRNA in VCAM-1-positive, but not VCAM-1-negative, bone marrow cells. Establishing specificity of RANKL gene expression, I $B \alpha$ mRNA, the product of another TNF target gene, was enhanced in both cell populations. Human mesenchymal stem cells (hMSCs), treated with TNF in osteogenic conditions, also expressed an abundance of RANKL mRNA, induction of which maximizes at $1 \mathrm{ng} / \mathrm{ml}$ of the cytokine (Figure $1 \mathrm{~B}$ ).

TNF-induced RANKL expression is mediated by IL-1. IL-1, another major cytokine produced in inflammatory sites, activates the RANKL gene in stromal cells (11) (Figure 2, A-C) and in other circumstances is downstream of TNF $(4,16)$. In this regard, TNFinduced RANKL mRNA synthesis was substantially inhibited in the presence of IL-1 receptor antagonist (IL-1Ra) in primary murine marrow stromal cells and hMSCs, which suggests that the 2 cytokines may be acting in sequence (Figure 2, A and B). In keeping with this posture, TNF-induced RANKL gene expression is also blunted in stromal cells derived from type I IL-1 receptor-deficient (IL-1RI-deficient) mice (Figure 2C). Confirming specificity of this latter event, absence of IL-1RI did not affect $1,25(\mathrm{OH})_{2} \mathrm{D}_{3}$-induced RANKL mRNA, and TNF-stimulated synthesis of $I \kappa B \alpha$ mRNA also remained robust. Similar to its pre- dominant expression by murine macrophages (17), mouse stromal cells exposed to TNF principally produced IL-1 $\alpha$ as opposed to IL-1 $\beta$ (data not shown).

IL-1RI is the functional signaling receptor for IL-1 (18), and its gene is expressed by marrow stromal cells under the influence of TNF. Again attesting to the sequential relationship of TNF and IL-1 in stromal cell signaling is the fact that TNF enhances IL-1 (Figure 3A) and both cytokines induce IL-1RI (Figure 3B). These observations are consistent with a model in which TNF-induced RANKL synthesis by stromal cells reflects an autocrine/paracrine event involving enhanced expression of IL-1 and its receptor.

To determine the effects of TNF and IL-1 on RANKL expression by stromal cells in the functional setting of osteoclastogenesis, we turned to an in vitro model in which osteoclasts are generated by coculture of marrow stromal cells and macrophages in the absence of exogenous RANKL. Because TNF, in the presence of permissive levels of RANKL, induces osteoclastogenesis by direct stimulation of macrophages (9), we used macrophages lacking both TNF receptors. In any circumstance, IL-1 alone, like low-dose TNF, was incapable of inducing osteoclast formation (Figure 4). Furthermore, high-dose TNF-induced osteoclastogenesis is blunted approximately $50 \%$ by IL-1Ra. The same results were obtained when osteoclastogenic cultures contained stromal cells derived from IL-1RI-deficient mice.

Having established IL-1 dependence of TNF-induced osteoclast formation in vitro, we asked whether the same occurs in vivo. Thus, vehicle or TNF ( 0.75 or $1.5 \mu \mathrm{g}$ ) was injected subcutaneously at the base of the skull daily for 5 days into WT or IL-1RI-defi-

\section{Figure 2}

TNF-induced RANKL gene expression is mediated by IL-1. (A and C) VCAM-1-positive WT or IL-1RI-deficient (IL-1RI KO) murine marrow cells or (B) hMSCs were exposed to designated cytokines or 1,25 -dihydroxyvitamin $D\left(10^{-8} \mathrm{M}\right)$ for 24 hours. In some circumstances TNF was added conjointly with IL-1Ra. RNA was extracted and subjected to RT-PCR. Numbers in parentheses represent concentrations in $\mathrm{ng} / \mathrm{ml}$.

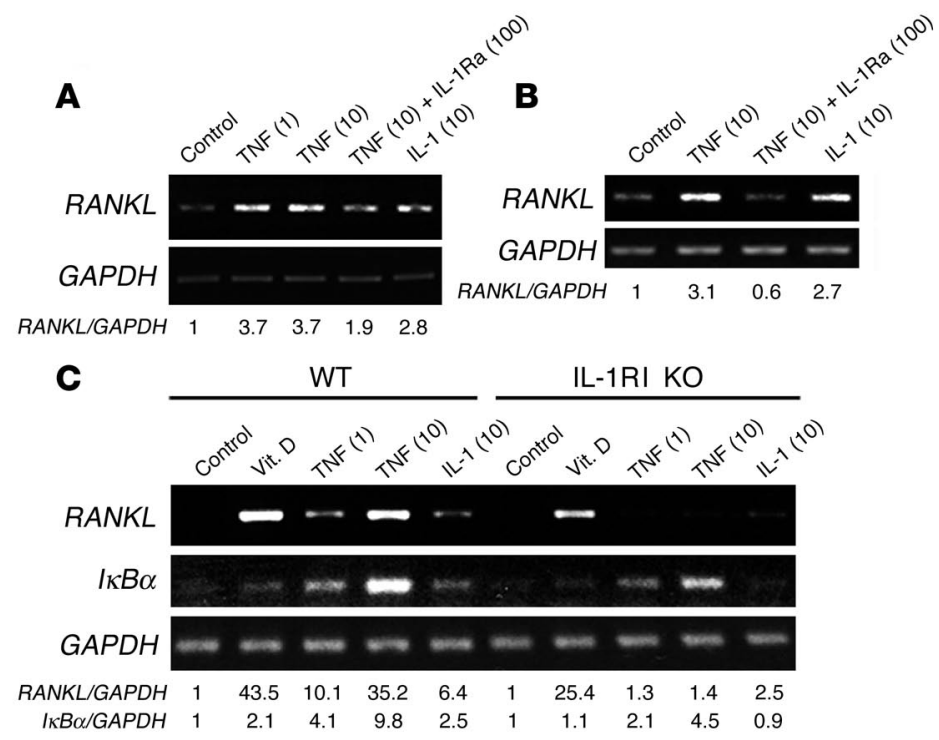


A

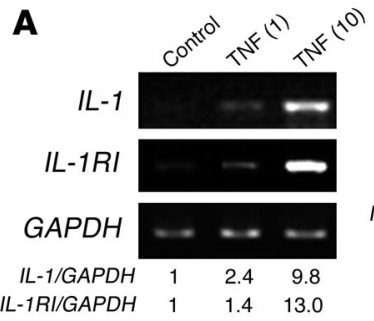

B

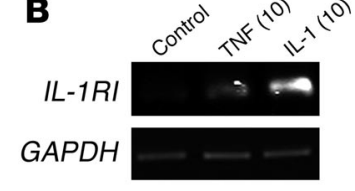

IL-1RI/GAPDH $\quad 1 \quad 5.5 \quad 23.1$ cient mice. Tartrate-resistant acidic phosphatase-stained (TRAPstained) sections of calvariae were prepared when the mice were sacrificed and osteoclast number histomorphometrically determined. TNF at both doses substantially increased osteoclastogenesis in WT mice (Figure 5). Reflecting what we observed in vitro, the magnitude of osteoclast recruitment induced by the inflammatory cytokine in mice lacking IL-1RI was approximately $50 \%$ that of their WT counterparts. Thus, IL-1 plays a central role in TNF targeting of stromal cells in the osteoclastogenic process. Alternatively, the persistence of stimulated osteoclastogenesis - albeit reduced by more than $50 \%$ in the presence of IL-1Ra or IL-1RI-deficient stromal cells in vitro and to the same extent in mice lacking the functional IL-1 receptor in vivo - indicates that TNF also affects osteoclast recruitment in a manner independent of IL- 1 receptor activation.

IL-4 inhibits TNF- and IL-1-induced RANKL expression via $338 M A P K$. IL-4 is an abundant antiinflammatory cytokine produced mainly by activated $\mathrm{T}$ lymphocytes. We previously demonstrated that this immunoregulatory protein blunts osteoclastogenesis by selectively inhibiting RANKL signaling in osteoclast precursors (19). To determine whether this molecule also plays a role in TNF-induced RANKL expression, we pretreated primary murine stromal cells

\section{Figure 3}

TNF induces IL-1 and IL-1RI in stromal cells. (A) VCAM-1-positive murine marrow cells and (B) hMSCs were exposed to vehicle (Control) or indicated cytokines for 24 hours. Numbers in parentheses represent concentrations in $\mathrm{ng} / \mathrm{ml}$. Total RNA was extracted and analyzed by RT-PCR. and hMSCs with IL-4 and then stimulated them with either TNF or IL-1. Pretreatment with IL-4 completely attenuated TNF- or IL-1-induced RANKL in both cell types (Figure 6).

IL-4 enhances IL-1-stimulated signaling in primary marrow macrophages (19). We asked whether this cytokine exerts similar effects in stromal cells by pretreating them with IL-4, then exposing them to IL-1. We subjected the cells to Western blot analysis to assess phosphorylation of p38, JNK, ERK, and IкB $\alpha$. To assure clinical relevance of these signaling experiments, we performed them with hMSCs. While IL-1-mediated phosphorylation of JNK, ERK, and IкB $\alpha$ was unaffected by prior IL-4 exposure, p38 activation was arrested with time (Figure 7).

These findings suggest that TNF-induced IL-1 mediates RANKL expression by marrow stromal cells via p38 MAPK. To explore this hypothesis, we turned to SB203580, an agent that does not affect p38 phosphorylation but specifically inhibits its capacity to activate downstream signaling molecules. In this regard, SB203580 dose-dependently inhibited phosphorylation of the known p38 target, ATF-2, whether induced by TNF (Figure $8 \mathrm{~A}$ ) or IL-1 (Figure 8B). Furthermore, incubation of hMSCs with SB203580, 1 hour prior to TNF or IL-1 exposure progressively suppressed $R A N K L, I L-1$, and $I L-1 R I$ mRNA expression (Figure

\section{Figure 4}

IL-1 mediates TNF-induced osteoclastogenesis in vitro. WT or IL-1RI-deficient, VCAM-1-positive murine marrow stromal cells were cocultured with bone marrow macrophages derived from p55/p75 TNF receptor-deficient mice in the presence of only the indicated cytokines. IL-1Ra (100 $\mathrm{ng} / \mathrm{ml}$ ) was added to half the cultures containing WT stromal cells. Medium was changed every 3 days. (A) After 7 days, the cells were stained for TRAP activity and (B) the number of multinucleated TRAP-positive cells determined. Values are mean \pm SD for 6 determinations per group ( ${ }^{\star *} P<0.01$ vs. WT). OC, osteoclasts.
A
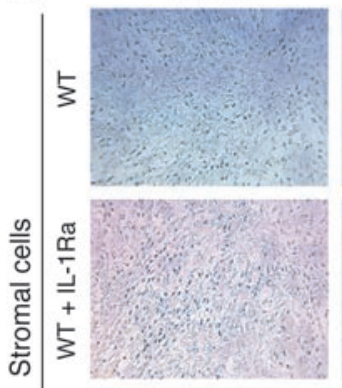

TNF (1 ng/ml)

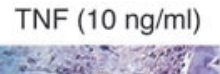

\section{$\mathrm{IL}-1(10 \mathrm{ng} / \mathrm{ml})$}
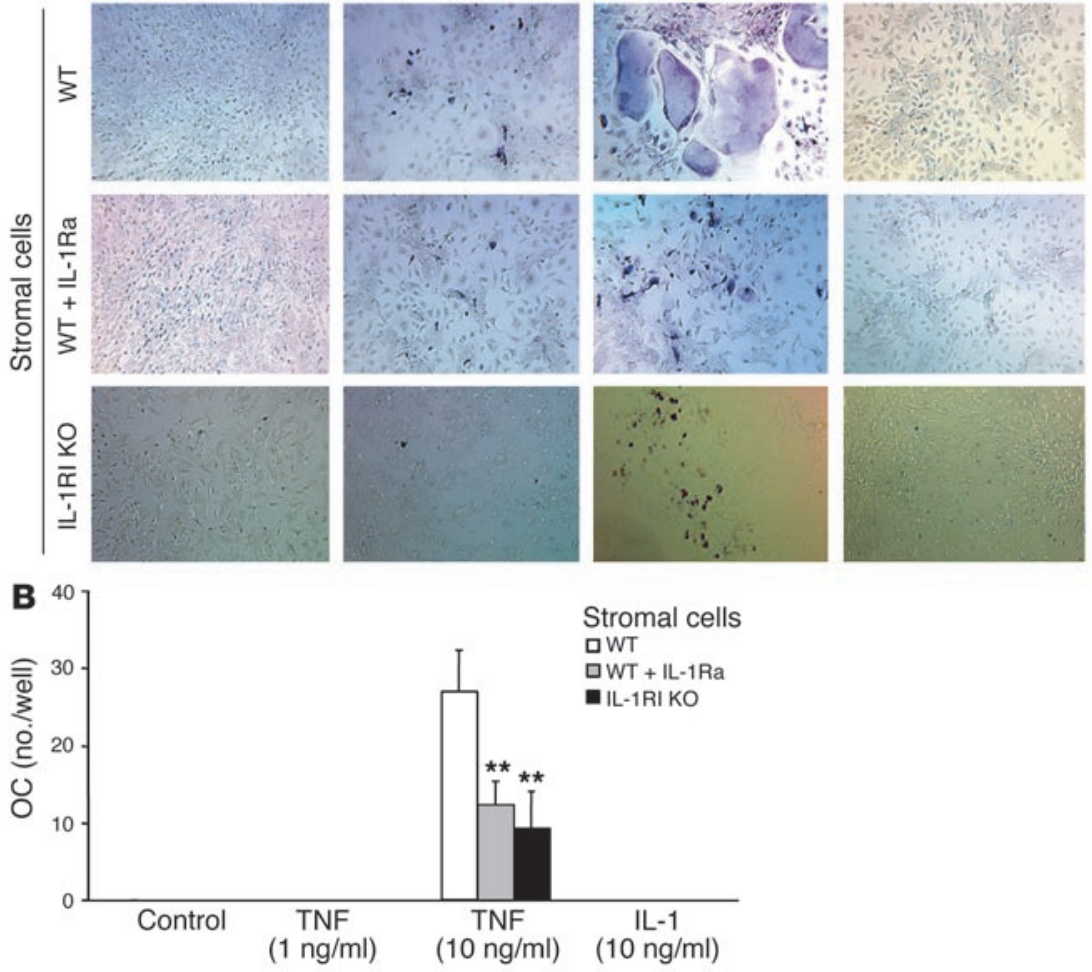

Stromal cells

aWT

aWT + IL-1Ra

- IL-1RI KO 
A
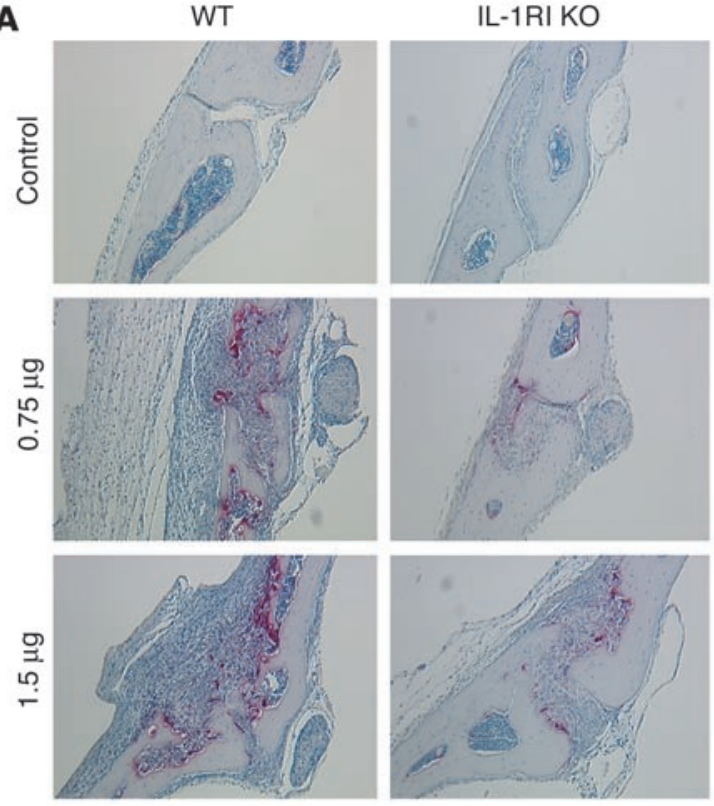

B

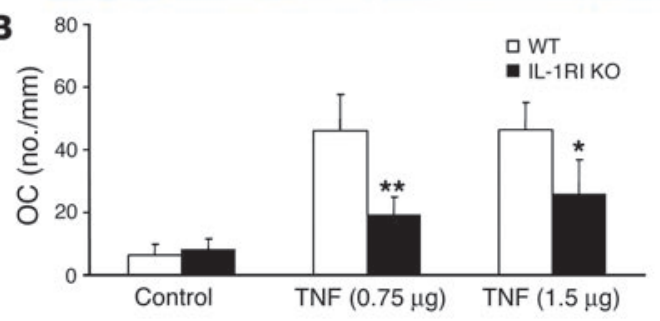

8C). Thus, p38 is a major pathway by which TNF and IL- 1 stimulate RANKL production by stromal/osteoblastic cells.

IL-1 enhances RANKL-induced osteoclastogenesis. The data presented thus far establish a central role for IL-1 in TNF-induced osteoclastogenesis via enhanced RANKL production by marrow stromal cells. TNF also stimulates osteoclast differentiation by directly targeting mononuclear osteoclast precursors in the presence of minimal levels of RANKL $(9,15)$. In fact, TNF prompts IL-1 gene expression in marrow macrophages in a p38-dependent manner (Figure 9). To determine whether IL-1 also has the capacity to directly promote osteoclast precursor differentiation, we cultured isolated bone marrow macrophages with increasing amounts of the cytokine in the presence or absence of a subosteoclastogenic concentration of RANKL. Similar to TNF, IL-1 alone, at any concentration, did not recruit osteoclasts but dose-dependently did so in the presence of minimal RANKL (Figure 10). To exclude the possibility that the IL-1-inductive effects represent stimulated expression of TNF, we repeated the experiment using macrophages derived from mice lacking both TNF receptors. Because these latter results mirror those of the experiment using WT macrophages, we conclude that IL-1 directly stimulates osteoclast precursor differentiation downstream of TNF.

To determine the stage of osteoclast differentiation at which IL-1 exerts its direct effect, we added the cytokine once to cultures of WT macrophages containing a subosteoclastogenic concentration of RANKL. The single addition of IL-1 occurred in different wells on day 0 through day 4 , followed by TRAP staining on day 5 . IL-1, added at any time, prompted substantial osteoclastogenesis (Figure 11, A and B). Concurrently, IL-1RI mRNA was upregulated

\section{Figure 5}

IL-1 mediates TNF-induced osteoclastogenesis in vivo. WT and IL-1RIdeficient mice were subcutaneously injected daily for 5 days at the base of the skull with vehicle or 0.75 or $1.5 \mu \mathrm{g}$ TNF. The animals were sacrificed on day 6 and TRAP-stained histological sections of calvariae histomorphometrically analyzed for osteoclast number. (A) Representative histological sections. (B) Histomorphometric analysis of osteoclast number ( ${ }^{*} P<0.05,{ }^{* \star} P<0.01$ vs. WT).

in the late phase of RANKL-induced osteoclastogenesis, whereas expression of IL-1RII, a decoy receptor for IL-1, was reduced by RANKL stimulation (Figure 11C). Like RANKL, TNF and IL-1 also promoted $I L-1 R I$ gene expression predominantly after marrow macrophages had committed to the osteoclast phenotype (Figure 12A). Similar to TNF- and IL-1-stimulated RANKL gene expression by stromal cells (Figure 8), inhibition of p38 MAPK arrested the capacity of the 3 cytokines to enhance IL-1RI mRNA in osteoclast precursors (Figure 12B). Finally, TNF-stimulated IL-1RI gene expression was unaltered by IL-1Ra (Figure 12C). Thus a component of TNF-induced osteoclastogenesis probably involves stimulated IL-1RI expression by committed osteoclast precursors. This event involves 2 distinct signaling pathways, 1 of which is mediated by, and the other independent of, IL-1.

\section{Discussion}

IL-1 promotes multinucleation of osteoclast precursors and enhances the capacity of the mature polykaryon to resorb bone (20). The fact that, in other circumstances, IL-1 is a downstream effector of TNF prompted us to ask whether the same holds true regarding stimulated RANKL synthesis by marrow stromal cells, and we found this to be the case. To determine whether the sequential action of TNF and IL-1 is manifested by enhanced osteoclast recruitment, we turned to purified populations of TNF-exposed marrow macrophages and stromal cells. We restricted TNF targeting to the stromal component by using marrow macrophages derived from TNF receptor-deficient mice. First, we prevented IL-1 from activating its receptor on both stromal

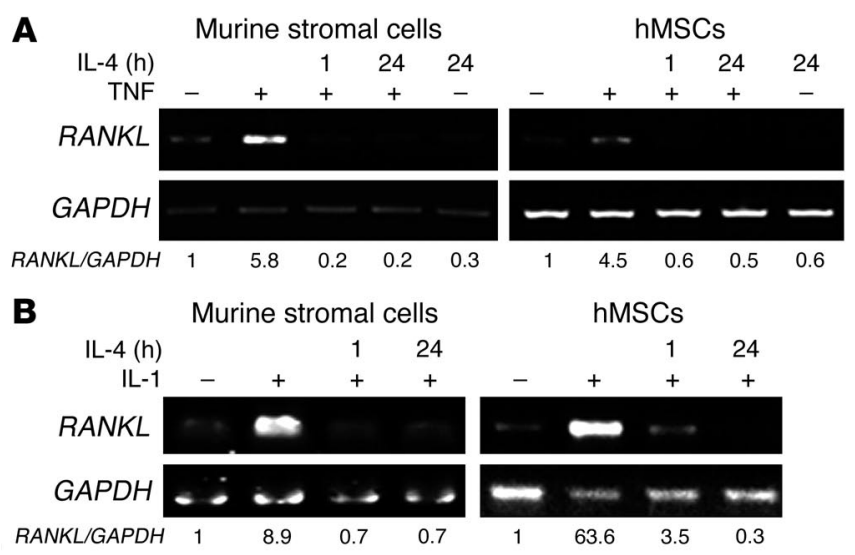

\section{Figure 6}

IL-4 inhibits TNF- or IL-1-induced RANKL expression. VCAM-1-positive murine marrow cells or hMSCs were pretreated with IL-4 (10 ng/ $\mathrm{ml}$ ) for indicated periods, followed by exposure to vehicle (-) or (A) TNF (10 ng/ml) or (B) IL-1 (10 ng/ml) for 24 hours. RNA was extracted and analyzed by RT-PCR. 


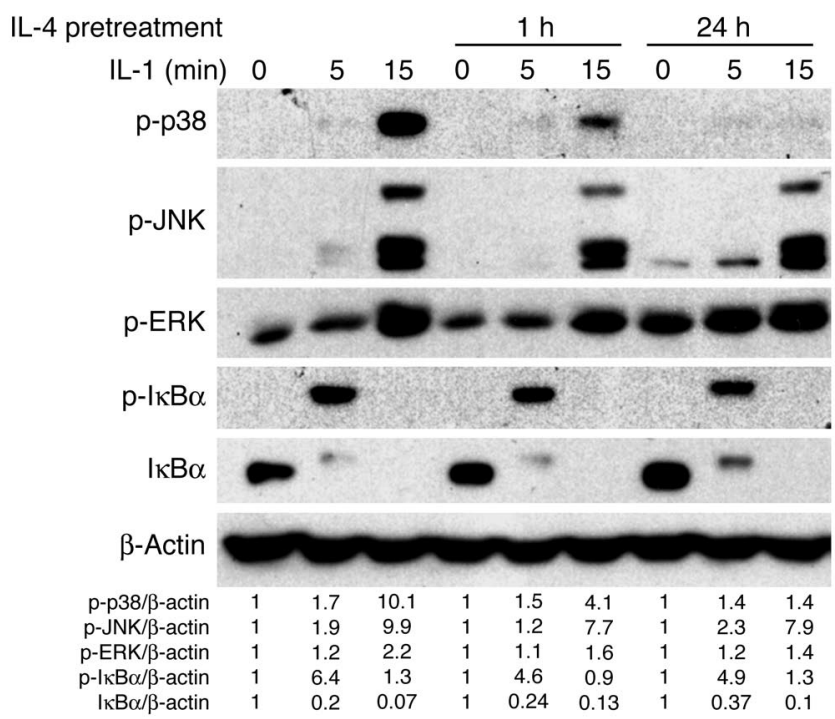

cells and osteoclast precursors with IL-1Ra. Second, we modified the model by using stromal cells lacking the functional IL-1 receptor. These in vitro experiments demonstrated that elimination of only IL-1-responsive stromal cells blunts TNF-induced osteoclastogenesis as effectively as inhibiting IL-1 signaling in both cellular constituents of the culture.

Regardless of the manner of IL-1 inhibition, however, TNF remained capable of substantial, albeit abridged, osteoclastogenesis. Importantly, TNF-induced osteoclast recruitment in vivo in mice lacking IL-1RI mirrors that occurring in both in vitro models. These data, in concert, establish that TNF exerts

\section{Figure 7}

IL-4 inhibits IL-1-mediated p38 MAPK activation. hMSCs were pretreated without or with IL-4 (10 ng/ml) for 1 or 24 hours. Cells were then exposed to IL-1 $(10 \mathrm{ng} / \mathrm{ml})$ for the indicated periods. Cells were lysed, fractionated by SDS-PAGE, and analyzed by immunoblot using antibodies recognizing total and phosphorylated signaling molecules. $\beta$-actin served as loading control. p-, phospho-.

its osteoclastogenic effect via IL-1-dependent and -independent signaling pathways and that the IL-1-mediated effect involves, at least in part, RANKL-producing stromal cells. This conclusion is buttressed by the fact that osteoclastogenesis stimulated by IL-1, like that stimulated by $\operatorname{TNF}(9,15)$, is RANKL dependent. On the other hand, while TNF fails to meaningfully stimulate RANKL expression by IL-RI-deficient stromal cells, the inflammatory cytokine promotes osteoclastogenesis, although attenuated, in the presence of these mutated stromal cells and in mice from which they are derived. This observation is in keeping with the exquisite synergy enjoyed by TNF and RANKL and the fact that constitutive levels of the RANKL are sufficient to enable TNF's osteoclastogenic properties (9).

Despite IL-1's capacity to induce RANKL gene expression and the synergistic relationship that also exists between the 2 cytokines, IL-1 alone is incapable of promoting osteoclast formation in macrophage/stromal cell coculture. While the explanation for this seeming discrepancy remains unexplained, it may reflect the nature of the coculture system. Support for this posture comes from a similar failure of low-dose TNF to stimulate osteoclast formation in such cultures while doing so in conditions in which pure populations of bone marrow macrophages are exposed to subosteoclastogenic concentrations of $\operatorname{RANKL}(9,15)$.

\section{Figure 8}

p38 MAPK mediates TNF- and IL-1-induced RANKL and IL-1RI expression. hMSCs were pretreated with SB203580 at the indicated concentrations overnight before exposure to vehicle (-) or (A) TNF (10 ng/ml) or (B) IL-1 (10 ng/ml) for 15 minutes. Cells were lysed and fractionated by SDS-PAGE, and activation of signaling molecules determined by immunoblot. ATF-2, activating transcription factor-2. (C) hMSCs were pretreated with decreasing concentrations of SB203580 for 30 minutes before exposure to PBS, TNF (10 $\mathrm{ng} / \mathrm{ml})$, or IL-1 $(10 \mathrm{ng} / \mathrm{ml})$ for 24 hours. RNA was extracted and analyzed by RT-PCR.
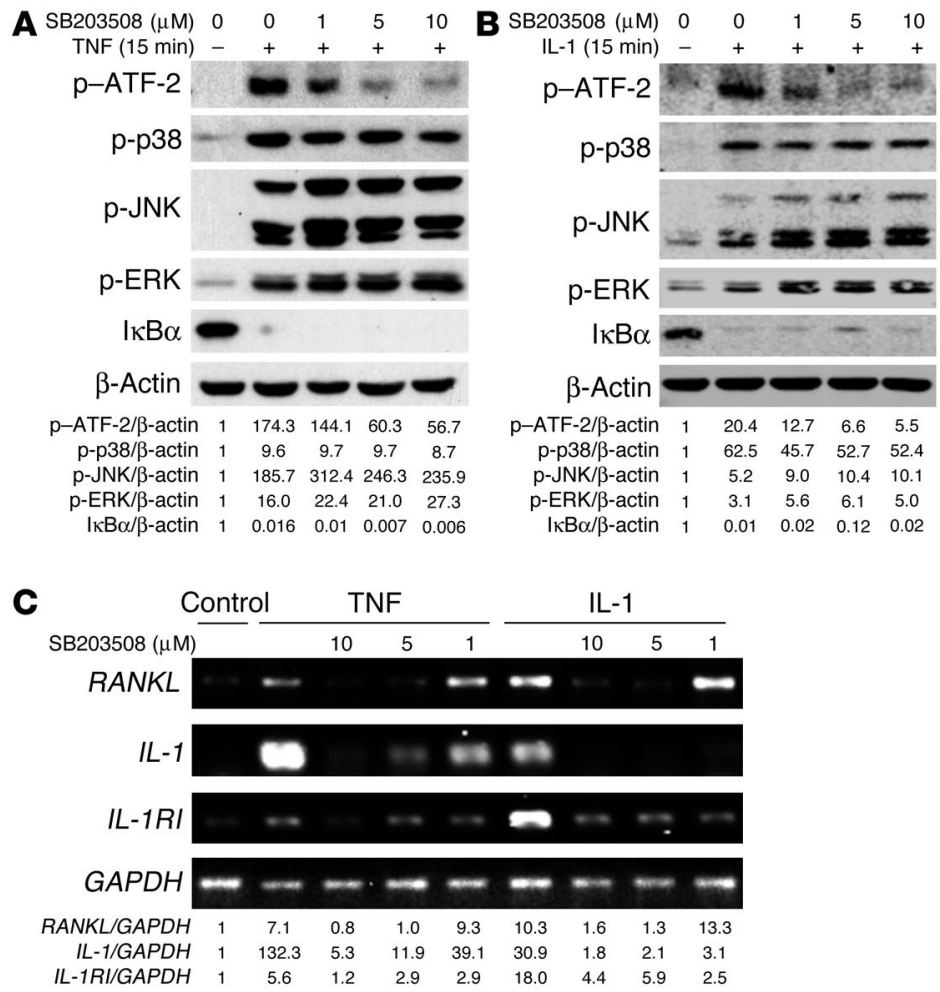


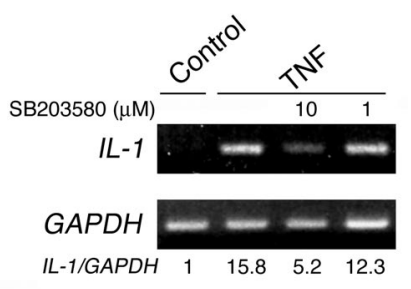

The present study establishes that the antiosteoclastogenic cytokine IL-4 blunts TNF- or IL-1-stimulated RANKL expression by marrow stromal cells. IL-4 also selectively dampens IL-1-mediated activation of $\mathrm{p} 38$, which promotes marrow macrophages to undergo osteoclast differentiation $(21,22)$. The importance of p38 in inflammatory osteoclastogenesis is underscored by the fact that its inhibition arrests cytokine production and bone loss in arthritic animals (23-25). The capacity of a specific p38 inhibitor to arrest IL-1- or TNF-induced RANKL and IL-1 gene expression by stromal cells is consistent with sequential activation of the 2 inflammatory cytokines in RANKL induction and, thus, the osteoclastogenic process.

$\mathrm{TNF}$, in the presence of subosteoclastogenic concentrations of RANKL, directly induces marrow macrophages to undergo osteoclast differentiation, and we found the same was true regarding IL-1. However, unlike TNF, whose capacity to prompt optimal osteoclast formation depends upon the presence of IL-1, this latter cytokine (i.e., IL-1) generates osteoclasts independently of TNF responsivity by marrow macrophages. Thus, whereas IL-1 is downstream of TNF in the osteoclastogenic process, the reciprocal does not occur.

These and our previous results suggest a model of optimal TNF-induced osteoclastogenesis in which the cytokine is recognized by its p55 receptor on stromal cells and osteoclast precursors (Figure 13). TNF, signaling through p38 MAPK, induces stromal cell expression of IL-1, which in turn upregulates its own receptor. Occupancy of nowabundant IL-1RI similarly activates p38, which promotes RANKL production. In macrophages, TNF enhances RANK expression (15) and synthesis of IL-1 (26), whose functional receptor is, in turn, upregulated by the same 3 cytokines (each via p38), while its decoy receptor, IL-1RII, is suppressed by RANKL. In the case of TNF, IL-1RI upregulation occurs via a combination of IL-1-dependent and -independent signaling pathways. IL-1, interact-

\section{Figure 10}

IL-1 directly induces osteoclast precursor differentiation. (A) WT or p55/p75 TNF receptor-deficient (TNFR $\mathrm{KO})$ murine marrow macrophages were cultured in human recombinant $\mathrm{M}-\mathrm{CSF}(50 \mathrm{ng} / \mathrm{ml})$ without or with RANKL and/or IL-1 at the indicated concentrations $(\mathrm{ng} / \mathrm{ml})$ at the initiation of culture. TRAP stain was performed on day 5. (B) Number of osteoclasts in cultures illustrated in panel $\mathbf{A}\left({ }^{*} P<0.05,{ }^{* *} P<0.01\right.$, $\# P<0.001$ vs. no addition).

\section{Figure 9}

TNF stimulates IL-1 gene expression by osteoclast precursors in a p38-dependent manner. WT murine marrow macrophages were pretreated with decreasing concentrations of SB203580 for 30 minutes before exposure to PBS (Control) or TNF $(10 \mathrm{ng} / \mathrm{ml})$ for 24 hours. Numbers in parentheses represent concentrations in $\mathrm{ng} / \mathrm{ml}$. RNA was extracted and analyzed by RT-PCR.

ing with its receptor on osteoclast precursors, in conjunction with RANKL, directly induces these cells to commit to the osteoclast phenotype. Given the synergistic relationship of the 2 cytokines, the dependence of TNF on stimulated, as compared with constitutive, expression of RANKL diminishes with enhanced joint inflammation. Thus, the interdependency of TNF, RANKL, and IL-1 in
RANKL

A
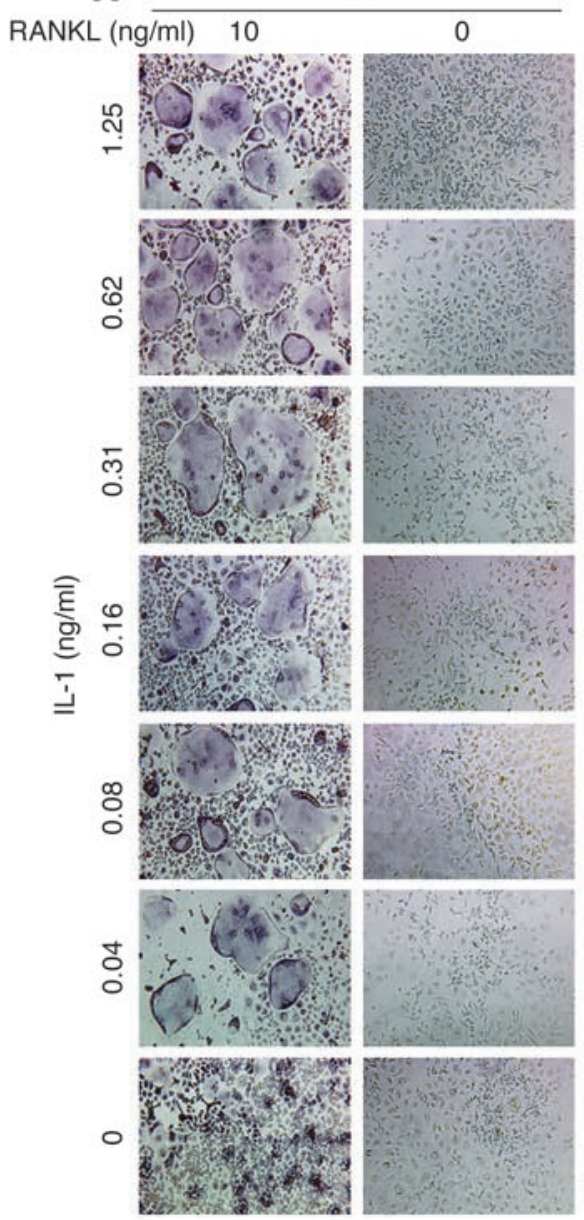

B

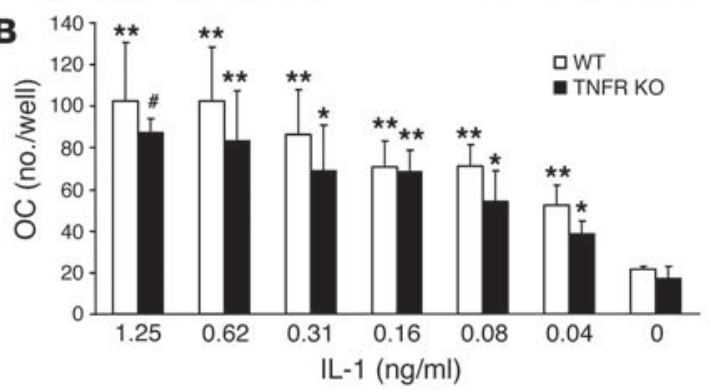

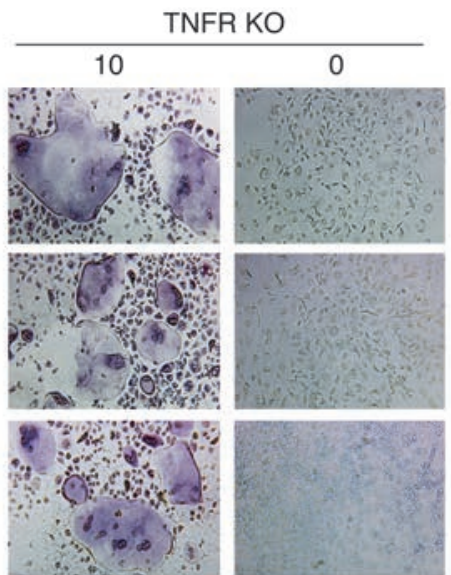
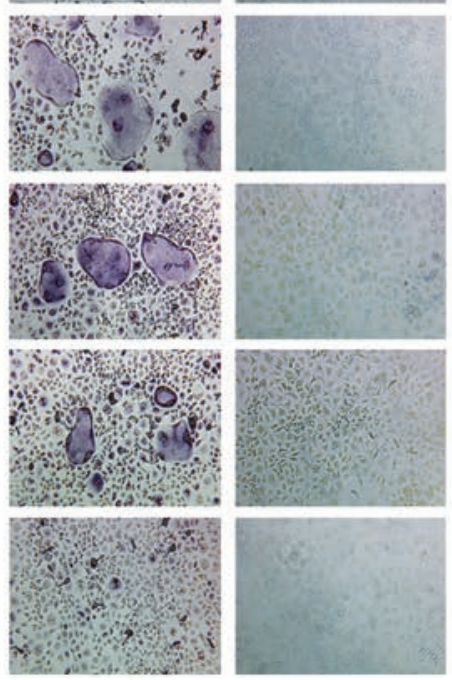


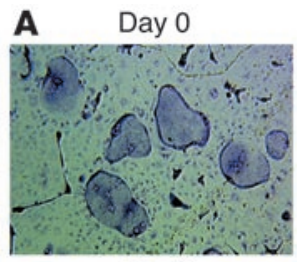

Day 3

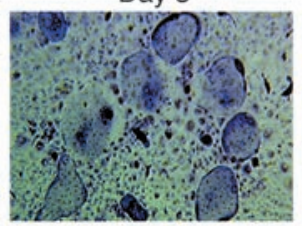

B
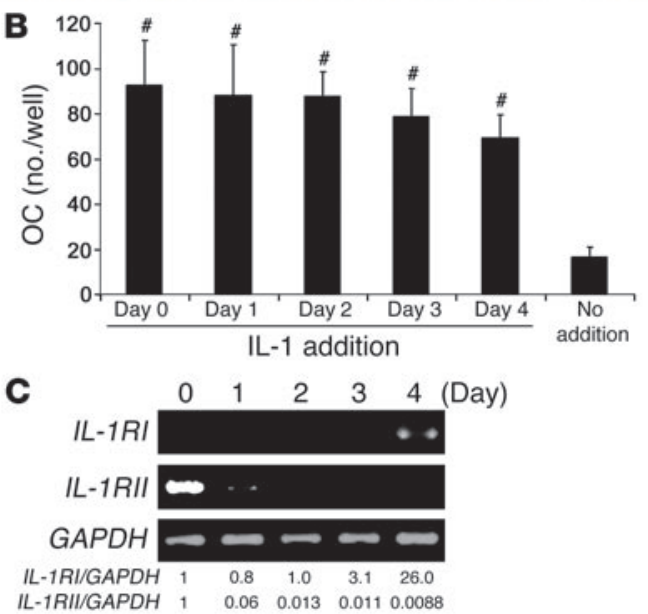

the generation of osteoclasts lends credence to the observation that combined blockade is most effective in preventing the periarticular bone loss of inflammatory arthritis (14).

\section{Methods}

Reagents. MACS separation columns, MACS magnet, and anti-rat IgG microbeads were purchased from Miltenyi Biotec GmbH. Rat antimouse CD106 (VCAM-1) monoclonal antibody was from BD Biosciences

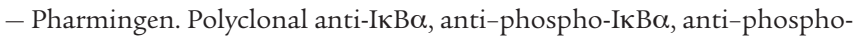
JNK, anti-phospho-p38, and anti-phospho-ERK antibodies were from Cell Signaling Technology. Protease inhibitor mixtures and SB203580 were from Calbiochem. The Coomassie Plus protein assay reagent and enhanced chemiluminescence kits were obtained from Pierce Biotechnology Inc. Recombinant M-CSF, IL-4, TNF- $\alpha$, IL- $1 \alpha$, and IL-1Ra were from R $\&$ D Systems. Murine RANKL was expressed in our laboratory as described pre-

\section{Figure 12}

TNF and IL-1 induce IL-1RI expression in committed preosteoclasts in a p38-dependent manner. (A) Freshly isolated bone marrow macrophages (BMMs) and the same cells exposed to M-CSF plus RANKL for 3 days (preosteoclasts [pre-OCs]) were washed with PBS and then treated with TNF- $\alpha$, IL-1, or RANKL for 24 hours. Total RNA was extracted with time and analyzed by RT-PCR for IL-1RI. (B) Preosteoclasts were pretreated with decreasing concentrations of SB203580 for 30 minutes before exposure to PBS (Control) or TNF (10 ng/ml), IL-1 (10 ng/ml), or RANKL (100 ng/ml) for 24 hours. RNA was extracted and analyzed by RT-PCR. (C) Preosteoclasts were treated with TNF $(10 \mathrm{ng} / \mathrm{ml})$ with or without IL-1Ra $(100 \mathrm{ng} / \mathrm{ml})$ for 24 hours. Numbers in parentheses represent concentrations in $\mathrm{ng} / \mathrm{ml}$. RNA was extracted and analyzed by RT-PCR.

\section{Figure 11}

IL-1 induction of osteoclast precursor differentiation occurs within a broad temporal window. (A) WT marrow macrophages were cultured in the presence of M-CSF $(50 \mathrm{ng} / \mathrm{ml})$ and RANKL $(10 \mathrm{ng} / \mathrm{ml})$. IL-1 $(1.25 \mathrm{ng} / \mathrm{ml})$ or vehicle was added only once to each well. IL-1 addition occurred on sequential days from day 0 through day 4. TRAP stain was performed on day 5. (B) Osteoclast number in each culture condition described in $\mathbf{A}$ ( $\# P<0.0001$ vs. no IL-1 addition). (C) WT marrow macrophages were cultured in the presence of M-CSF $(50 \mathrm{ng} / \mathrm{ml})$ plus RANKL $(100 \mathrm{ng} / \mathrm{ml})$. Total RNA was extracted with time and analyzed by RT-PCR for abundance of $I L-1 R I$ and $I L-1 R I /$ mRNA.

viously (27). $\beta$-actin antibody and all other chemicals were obtained from Sigma-Aldrich.

Mice. WT C57BL/6 mice, IL-1RI-deficient mice, and p55 and p75 TNF receptor-deficient mice were purchased from The Jackson Laboratory. Mice deficient in both $\mathrm{p} 55$ and $\mathrm{p} 75 \mathrm{TNF}$ receptors were generated by crossing $\mathrm{p} 55$ and $\mathrm{p} 75 \mathrm{TNF}$ receptor-deficient mice. All animal experimentation was approved by the Animal Studies Committee of Washington University School of Medicine.

Cell culture. Murine marrow cells were extracted from whole bone marrow of 4- to 6-week-old male mice and incubated in tissue culture dishes in $\alpha$-MEM supplemented with $10 \%$ heat-inactivated FBS at $37^{\circ} \mathrm{C}$ in $5 \% \mathrm{CO}_{2}$. After 24 hours in culture, M-CSF-dependent macrophages were obtained by culturing the nonadherent marrow cells in the presence of M-CSF in tissue culture dishes. Bone marrow stromal and osteoblastic cells were purified from the adherent cells after 5-7 days of culture by immunopurification antibody against VCAM-1. hMSCs were purchased from Cambrex

Corp. These cells were maintained in growth medium and switched to osteogenic differentiation medium (both from Cambrex Corp.) for 7 days before experimental stimulation.

Characterization of osteoclasts. Marrow macrophages were cultured in 96-well cell culture dishes in the presence of M-CSF and RANKL as well
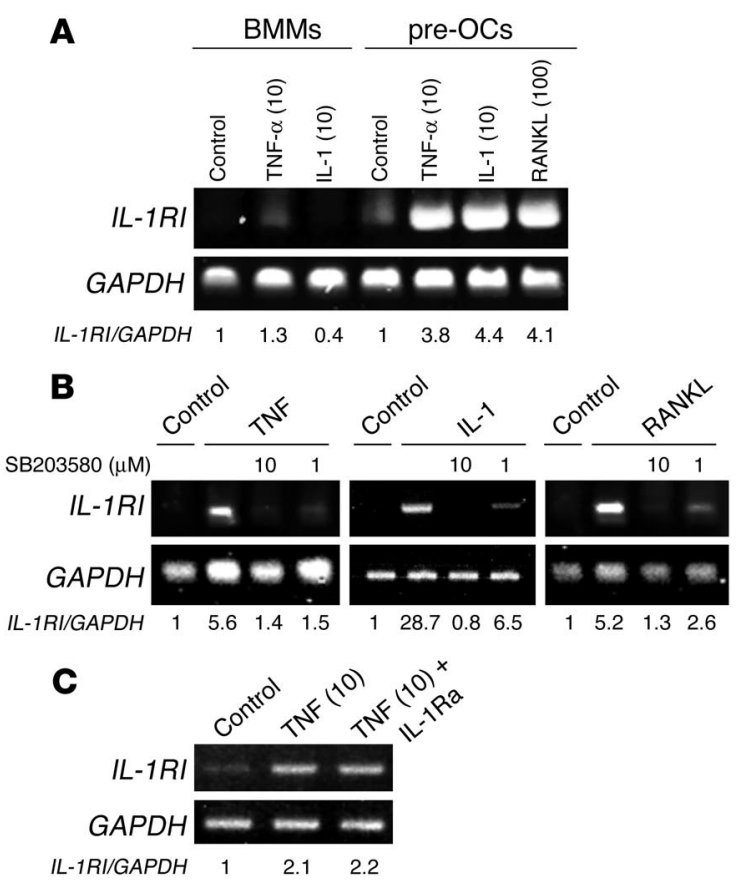


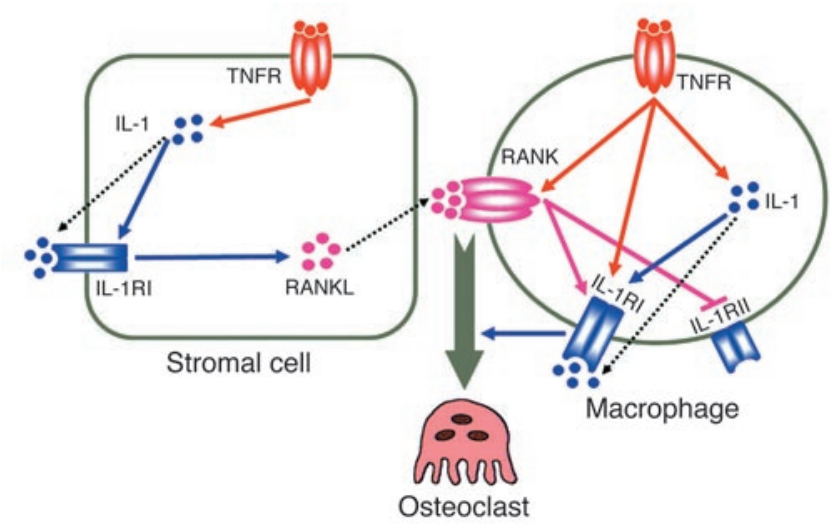

as other cytokines, as appropriate, and medium was changed on day 3, by which time they had differentiated into TRAP-expressing mononuculear cells (preosteoclasts). Coculture of marrow stromal cells and macrophages was performed in 96-well cell culture dishes in the absence and presence of cytokines as indicated in individual experiments, and medium was changed every 3 days. Osteoclast-like cells were characterized by staining for TRAP. $R N A$ extraction and amplification by reverse transcriptase-PCR. RNA was isolated using RNeasy kits and reverse-transcribed using $1 \mu \mathrm{g}$ of total RNA, $0.5 \mu \mathrm{g}$ of $(\mathrm{dT})_{15}$ primer, $20 \mathrm{U}$ of RNasin RNase inhibitor, $20 \mathrm{U}$ of avian myeloblastosis virus-reverse transcriptase, $\times 1$ avian myeloblastosis virus RT buffer (Promega Corp.), and $1 \mathrm{mM}$ deoxyribonucleoside triphosphate mix (Invitrogen Corp.) in a final volume of $20 \mu$ l. The reaction was carried out for 15 minutes at $42^{\circ} \mathrm{C}$ and for 5 minutes at $99^{\circ} \mathrm{C}$ and then cooled to $4^{\circ} \mathrm{C}$. The RT reaction products were further diluted to $100 \mu \mathrm{l}$, and 1 $\mu \mathrm{l}$ of each was applied to a $49-\mu \mathrm{l}$-volume PCR premix, containing $1 \mathrm{pmol}$ of each primer, $0.2 \mathrm{mM}$ dNTP mix, 5 units of Taq polymerase, $2 \mathrm{mM}$ $\mathrm{MgCl}_{2}$, and $\times 1$ PCR buffer (Invitrogen Corp.). PCR was carried out in a PCR Express thermal cycler (HYBAID) set for various cycles to monitor the linearity of the amplification, with annealing temperature at $60^{\circ} \mathrm{C}$. The oligonucleotide primers used were as follows: (a) murine GAPDH (5') 5'-ACTTTGTCAAGCTCATTTCC-3' and (3') 5'-TGCAGCGAACTTTATTGATG-3'; (b) human GAPDH (5') 5'-TGAAGGTCGGAGTCAACGGATTTGGT- $3^{\prime}$ and (3') 5'-CATGTGGGCCATGAGGTCCACCAC-3'; (c) IL-1RI (5') 5'-GGACTCCAGGATTCATCAGC-3' and ( $\left.3^{\prime}\right) 5^{\prime}$-AGGACATACGGCATATATAG-3'; (d) IL-1RII (5') 5'-GAGGTATAAGGGCGCCATAC-3' and $\left(3^{\prime}\right) 5^{\prime}$-GTAAGCAGCCGAGATAAACG-3'; and (e) IкB $\alpha\left(5^{\prime}\right) 5^{\prime}$-GCCTGGACTCCATGAAAGAC-3' and (3') 5'-CAAGTGGAGTGGAGTCTGCTGCAGGTTGTT- ${ }^{\prime}$. The primers for murine and human RANKL were purchased from R\&D Systems and those for human IL-1 $\alpha$ and murine IL- $1 \alpha / \beta$ from Maxim Biotech Inc., and PCR was performed following the manufacturer's instructions. All blots were densitometrically quantitated and the results expressed relative to control (arbitrarily delineated as 1) and normalized to GAPDH.

ELISA. Cells were incubated in a 12 -well plate and treated with stimuli as indicated, washed twice with ice-cold PBS, and then lysed by freezing and thawing 3 times in $250 \mu \mathrm{l}$ of $\alpha$-MEM without serum. Intracellular IL-1 $\alpha$ in

\section{Figure 13}

Mechanisms of TNF-induced osteoclastogenesis. As detailed in the text, TNF-induced osteoclastogenesis involves targeting of both stromal cells and osteoclast precursors. cell lysates was determined using an IL-1 $\alpha$ ELISA kit (R\&D Systems) following the manufacturer's instructions.

Immunoblotting. Cytokine-treated or control monolayers of hMSCs were washed twice with ice-cold PBS. Cells were lysed in the buffer containing $20 \mathrm{mM}$ Tris, pH 7.5, $150 \mathrm{mM} \mathrm{NaCl}, 1 \mathrm{mM}$ EDTA, 1 mM EGTA, 1\% Triton $\mathrm{X}-100,2.5 \mathrm{mM}$ sodium pyrophosphate, $1 \mathrm{mM} \beta$-glycerophosphate, $1 \mathrm{mM}$ $\mathrm{Na}_{3} \mathrm{VO}_{4}, 1 \mathrm{mM} \mathrm{NaF}$, and $\times 1$ protease inhibitor mixture. Fifty micrograms of cell lysates were boiled in the presence of SDS sample buffer $(0.5 \mathrm{M}$ Tris$\mathrm{HCl}, \mathrm{pH} 6.8,10 \%$ [wt/vol] SDS, 10\% glycerol, $0.05 \%$ [wt/vol] bromphenol blue) for 5 minutes and subjected to electrophoresis on $8 \%$ SDS-PAGE. Proteins were transferred to PVDF membranes using a semi-dry blotter (Bio-Rad Laboratories) and incubated in blocking solution (5\% nonfat dry milk in TBS containing $0.1 \%$ Tween-20) for 1 hour to reduce nonspecific binding. Membranes were then exposed to primary antibodies overnight at $4{ }^{\circ} \mathrm{C}$, washed 3 times, and incubated with secondary goat anti-mouse or rabbit IgG HRP-conjugated antibody for 1 hour. Membranes were washed extensively, and enhanced chemiluminescence detection assay was performed following the manufacturer's directions. All blots were densitometrically quantitated and the results expressed relative to control and normalized to $\beta$-actin.

Statistics. Data are presented as mean $\pm \mathrm{SD}$. Statistical significance is calculated by Student's $t$ test.

\section{Acknowledgments}

This study was supported by NIH grants AR46852 and AR48812 (to F.P. Ross), AR32788, AR46523, AR48853, and DK56341 (Clinical Nutrition Research Unit) (to S.L. Teitelbaum).

Received for publication September 17, 2004, and accepted in revised form November 30, 2004.

Address correspondence to: Steven L. Teitelbaum, Department of Pathology, Washington University School of Medicine, Campus Box 8118, 660 South Euclid Avenue, St. Louis, Missouri 63110, USA. Phone: (314) 454-8463; Fax: (314) 454-5505; E-mail: teitelbs@wustl.edu.
1. Teitelbaum, S.L. 2000. Bone resorption by osteoclasts. Science. 289:1504-1508.

2. Ross, F.P. 2000. RANKing the importance of measles virus in Paget's disease. J. Clin. Invest. 105:555-558.

3. Locklin, R.M., Khosla, S., Turner, R.T., and Riggs, B.L. 2003. Mediators of the biphasic responses of bone to intermittent and continuously administered parathyroid hormone. J. Cell. Biochem. 89:180-190.

4. Feldmann, M., and Maini, R.N. 2001. Anti-TNF therapy of rheumatoid arthritis: what have we learned? Ann. Rev. Immunol. 19:163-196.

5. Gravallese, E.M. 2002. Bone destruction in arthritis. Ann. Rheum. Dis. 61(Suppl. 2):ii84-ii86.

6. Romas, E., Gillespie, M.T., and Martin, T.J. 2002. Involvement of receptor activator of NFkappaB ligand and tumor necrosis factor-alpha in bone destruction in rheumatoid arthritis. Bone. 30:340-346.

7. Redlich, K., et al. 2002. Osteoclasts are essential for TNF- $\alpha$-mediated joint destruction. J. Clin. Invest. 110:1419-1427. doi:10.1172/JCI200215582.
8. Ritchlin, C.T., Haas-Smith, S.A., Li, P., Hicks, D.G., and Schwarz, E.M. 2003. Mechanisms of TNF- $\alpha-$ and RANKL-mediated osteoclastogenesis and bone resorption in psoriatic arthritis. J. Clin. Invest. 111:821-831. doi:10.1172/JCI200316069.

9. Lam, J., et al. 2000. TNF- $\alpha$ induces osteoclastogenesis by direct stimulation of macrophages exposed to permissive levels of RANK ligand. J. Clin. Invest. 106:1481-1488.

10. Li, P., et al. 2004. RANK signaling is not required for 
TNFalpha-mediated increase in CD11(hi) osteoclast precursors but is essential for mature osteoclast formation in TNFalpha-mediated inflammatory arthritis. J. Bone Miner. Res. 19:207-213.

11. Hofbauer, L.C., et al. 1999. Interleukin-1 beta and tumor necrosis factor-alpha, but not interleukin-6, stimulate osteoprotegerin ligand gene expression in human osteoblastic cells. Bone. 25:255-259.

12. Dayer, J.M. 2002. Interleukin 1 or tumor necrosis factor-a: which is the real target in rheumatoid arthritis. J. Rheumatol. 29(Suppl. 65):10-15.

13. Arend, W.P. 2002. The mode of action of cytokine inhibitors. J. Rheumatol. 29(Suppl.):16-21.

14. Zwerina, J., et al. 2004. Single and combined inhibition of tumor necrosis factor, interleukin-1, and RANKL pathways in tumor necrosis factor-induced arthritis: effects on synovial inflammation, bone erosion, and cartilage destruction. Arthritis Rheum. 50:277-290.

15. Kitaura, H., et al. 2004. Marrow stromal cells and osteoclast precursors differentially contribute to TNF- $\alpha$ induced osteoclastogenesis in vivo. J. Immunol. 173:4838-4846.

16. Brennan, F.M., Chantry, D., Jackson, A., Maini, R., and Feldmann, M. 1989. Inhibitory effect of TNF alpha antibodies on synovial cell interleukin-1 production in rheumatoid arthritis. Lancet. 2:244-247.

17. Dinarello, C.A. 1996. Biologic basis for interleukin-1 in disease. Blood. 87:2095-2147.

18. Dunne, A., and O'Neill, L.A.J. 2003. The interleukin-1 receptor/toll-like receptor superfamily: signal transduction during inflammation and host defense [review]. Sci. STKE. 171:3.

19. Wei, S., Wang, M.W., Teitelbaum, S.L., and Ross, F.P. 2002. Interleukin-4 reversibly inhibits osteoclastogenesis via inhibition of NF-KB and MAP kinase signaling. J. Biol. Chem. 21:6622-6630.

20. Jimi, E., et al. 1999. Interleukin 1 induces multinucleation and bone-resorbing activity of osteoclasts in the absence of osteoblasts/stromal cells. Exp. Cell Res. 247:84-93.

21. Matsumoto, M., Sudo, T., Maruyama, M., Osada, H., and Tsujimoto, M. 2000. Activation of p38 mitogen-activated protein kinase is crucial in osteoclastogenesis induced by tumor necrosis factor. FEBS Lett. 486:23-28.

22. Li, X., et al. 2003. p38 mitogen-activated protein kinase is crucially involved in osteoclast differentia- tion but not in cytokine production, phagocytosis, or dendritic cell differentiation of bone marrow macrophages. Endocrinology. 144:4999-5005.

23. Badger, A.M., et al. 2000. Disease-modifying activity of SB 242235, a selective inhibitor of p38 mitogenactivated protein kinase, in rat adjuvant-induced arthritis. Arthritis Rheum. 43:175-183.

24. Nishikawa, M., et al. 2003. Prevention of the onset and progression of collagen-induced arthritis in rats by the potent $\mathrm{p} 38$ mitogen-activated protein kinase inhibitor FR167653. Arthritis Rheum. 48:2670-2681.

25. Badger, A., et al. 1996. Pharmacological profile of SB 203580, a selective inhibitor of cytokine suppressive binding protein/p38 kinase, in animal models of arthritis, bone resorption, endotoxin shock and immune function. J. Pharmacol. Exp. Ther. 279:1453-1461.

26. Ishida, N., et al. 2002. Large scale gene expression analysis of osteoclastogenesis in vitro and elucidation of NFAT2 as a key regulator. J. Biol. Chem. 277:41147-41156.

27. McHugh, K.P., et al. 2000. Mice lacking $\beta 3$ integrins are osteosclerotic because of dysfunctional osteoclasts. J. Clin. Invest. 105:433-440. 